\title{
Postmarketing pharmacokinetic and pharmacodynamic equivalence of generic and brand atenolol in Egypt
}

\author{
M.A. Badawi, ${ }^{1}$ E. Wagdy, ${ }^{2}$ M. Nasr, ${ }^{3}$ M.A. Etman, ${ }^{1}$ L.K. El-Khordagui ${ }^{1}$ and S.A.H. Khalil ${ }^{1}$
}

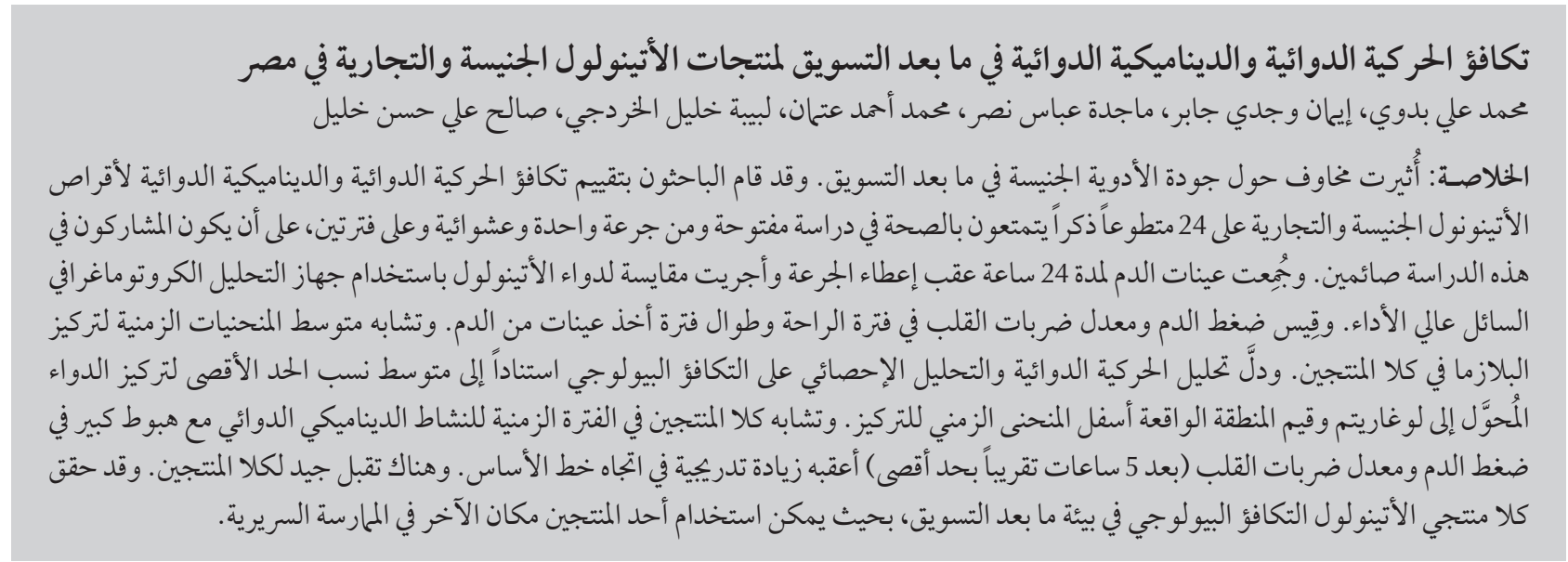

ABSTRACT Concerns have been raised regarding the postmarketing quality of generic drugs. This study assessed the pharmacokinetic and pharmacodynamic equivalence of generic and brand atenolol tablets in 24 healthy male volunteers in a single-dose, open, randomized, two-period crossover study under fasting conditions. Blood samples were collected for $24 \mathrm{~h}$ post dosing and assayed for atenolol using HPLC. Blood pressure and heart rate were measured at baseline and throughout blood sampling. The mean plasma concentration-time curves for both products were similar. Pharmacokinetic and statistical analysis indicated bioequivalence based on the mean ratios of log-transformed Cmax and AUC values. Both products had similar time courses of pharmacodynamic activity with a significant fall in blood pressure and heart rate (maximum after $\sim 5 \mathrm{~h}$ ) followed by a gradual increase towards baseline. Both products were well tolerated. Both atenolol products were bioequivalent in the postmarketing setting and can be used interchangeably in clinical practice.

Étude d'équivalence post-commercialisation des propriétés pharmacocinétiques et pharmacodynamiques de l'aténolol en générique et sous son nom de marque en Égypte

RÉSUMÉ La qualité post-commercialisation des médicaments génériques a suscité des inquiétudes. Nous avons évalué l'équivalence des propriétés pharmacocinétiques et pharmacodynamiques de comprimés d'aténolol génériques et de marque au cours d'une étude croisée en deux phases, randomisée, ouverte, sur une dose unique administrée à jeun chez 24 volontaires de sexe masculin en bonne santé. Des échantillons de sang ont été prélevés pendant les 24 heures suivant l'administration de la dose et la concentration en aténolol a été évaluée par chromatographie en phase liquide à haute performance. La tension artérielle et la fréquence cardiaque ont été mesurées au repos et à chaque prélèvement sanguin. L'aire sous la courbe de la concentration plasmatique moyenne sur un intervalle de temps défini était similaire pour les deux produits. L'analyse pharmacocinétique et statistique a indiqué une bioéquivalence reposant sur les rapports moyens de la concentration plasmatique maximale obtenue par calcul logarithmique et des valeurs de l'aire sous la courbe. Les deux produits avaient des évolutions temporelles similaires pour leur activité pharmacodynamique avec une diminution importante de la tension artérielle et de la fréquence cardiaque (maximum après 5 heures environ) suivie par un retour progressif vers les valeurs initiales. Les deux produits étaient bien tolérés. Les produits de marque et génériques de l'aténolol étaient bioéquivalents dans le cadre de l'étude post-commercialisation et peuvent être utilisés indifféremment en pratique clinique.

'Department of Pharmaceutics, Faculty of Pharmacy, Alexandria University, Alexandria, Egypt (Correspondence to L.K. El-Khordagui: Lakhalil@ gmail.com; Lakhalil@alexpharmacy.edu.eg).2Department of Internal Medicine, Medical Research Institute, Alexandria University, Alexandria, Egypt. ${ }^{3}$ Department of Pharmacology, Medical Research Institute, Alexandria University, Alexandria, Egypt.

Received: 18/09/12; accepted: 19/02/13 


\section{Introduction}

Generic or multisource medicines play a key role in ensuring the affordability and sustainability of healthcare in both developed and developing countries. According to the $\mathrm{WHO}$, multisource products that are both pharmaceutically equivalent and bioequivalent (thus therapeutically equivalent) are considered interchangeable [1]. In Egypt, the domestic consumption of drugs is dominated by the local generic industry, which has become a major export hub for the regional market $[2,3]$.

Although the quality of generics is assured initially for registration, sometimes concerns have been raised in different parts of the world about the therapeutic equivalence and interchangeability of branded and generic products in the postmarketing setting $[4,5]$. Such concerns have been rationalized by the notions that bioequivalence of two products, usually demonstrated using a single dose in normal healthy subjects does not imply comparable efficacy and tolerability in a patient population $[6,7]$ and that the process of approving generic medications is not as rigorous as that of brand-name medications. Poor therapeutic outcomes when patients are prescribed generic drugs and relapse of symptoms when patients are switched from brand-name drugs to generics, or from one generic to another, are usually the main cause of complaints $[7,8]$. Such complaints are supported by actual bioequivalence differences reported in the literature [9-11].

Atenolol is a $\beta$, selective antagonist that is used to treat essential uncomplicated hypertension. It is also used alone or in combination with other agents for management of angina pectoris and to reduce mortality and morbidity in secondary prevention after myocardial infarction. Atenolol was on the 2010 WHO Essential Medicines List [12] at the time of the study and one of the most commonly prescribed $\beta$-blockers worldwide [13]. Atenolol has one chiral centre and is available commercially as a racemate consisting of two enantiomers, the $(-)$ S isomer being the active form $[14,15]$. Atenolol is readily but incompletely absorbed after oral administration with widely variable peak plasma concentrations [15-18].

Several pharmacokinetic/pharmacodynamic studies in healthy subjects and clinical studies have indicated no superiority of the brand atenolol product Tenormin to its generic counterparts [16-19]. In Egypt, atenolol is marketed as Tenormin (Innovator), manufactured under license, and three generic drugs. These are widely prescribed for economic reasons but with some suspicion regarding the efficacy and interchangeability of the brand and generic products.

The aim of the present study was to perform an independent postmarketing reassessment of the bioequivalence and pharmacodynamic activity of a locally manufactured generic atenolol product in comparison to its brand counterpart. Such postmarketing monitoring is of importance to both the clinical community and national regulatory agencies regarding maintenance of quality of manufacturing procedures, safety of medicines for the general public, and informed planning of drug use.

\section{Methods}

\section{Study products}

The study products were 100-mg immediate-release, film-coated tablets of atenolol. The test product was a locally manufactured generic drug, Ateno (EIPICO, Egypt; batch No 086947, manufacturing date 10/2008 and expiry date 10/2011). The reference product was Tenormin (Astra Zeneca, Cairo, Egypt; under license, batch No 80130 , manufacturing date $08 / 2008$ expiry date $08 / 2013$ ).

\section{Study participants}

Twenty-four healthy male adult volunteers fulfilling the inclusion criteria were recruited: age 18-50 [mean 35.2 (SD 10.1)] years; body mass index 19.5 to < 30 [mean 26.7 (SD 4.1)] kg/m² and height 155-189 [mean 169.0 (SD $8.63)] \mathrm{cm}$. The volunteers underwent pre-study clinical assessments including medical history, physical examination, blood biochemistry, renal and liver functions, electrocardiography, and vital signs (blood pressure, heart rate and respiratory rate). Participants free from significant gastrointestinal, cardiac, renal, hepatic or haematological abnormalities and whose clinical laboratory data did not significantly deviate from reference values were enrolled. Clinical data and demographic information obtained by interview was documented in individual case report forms. Volunteers signed written informed consent after being informed by the clinical investigator of the purpose, procedure and risks of the study and their right to withdraw at any time. They were asked to abstain from taking drugs including over the counter and herbal medicines 2 weeks before and throughout the course of the study, and to report any adverse drug effects experienced during the study.

\section{Study design}

The study was a randomized, open-label, two-period crossover study with a 1-week washout period. The study was carried out in September 2009, in the inpatient ward facilities of the Medical Research Institute, Alexandria University, Alexandria, Egypt. The study protocol was approved by the Research Ethics Committee.

Following an overnight fast of at least $10 \mathrm{~h}$, participants were randomly administered a single dose (one 100 mg tablet) of the test or reference product with $200 \mathrm{~mL}$ water. The hands and mouth were checked to ensure that the tablets had been swallowed. No additional water or fluids were permitted until $2 \mathrm{~h}$ after drug intake. Participants were allowed to drink water $(200 \mathrm{~mL})$ after $3 \mathrm{~h}$. A standard meal was served at $\sim 6 \mathrm{~h}$ after drug administration, which consisted of grilled chicken, rice and 
vegetables, and an apple. Meal plans were identical in the two periods. Participants were not allowed to consume beverages and food containing caffeine from $48 \mathrm{~h}$ prior to drug administration until the end of the study. Participants did not engage in any strenuous activity at any time during blood sampling, to limit effects on gastrointestinal blood flow, blood pressure, and heart rate. Participants were under close medical supervision throughout the study.

Blood samples were collected from the antecubital vein in heparinized tubes before dosing and at the following times: $1,2,2.5,3,3.5,4,5,6,8,10,12$ and 24 h. Blood samples were centrifuged immediately after collection at $3500 \mathrm{rpm}$ for $10 \mathrm{~min}$. Plasma samples were transferred directly into plain plastic tubes and immediately stored at $-20^{\circ} \mathrm{C}$ pending analysis. A 7-day washout period was allowed between the two study periods.

Pharmacodynamic activity was monitored simultaneously by measuring blood pressure and heart rate at baseline and immediately prior to each blood sample for $24 \mathrm{~h}$. Tolerability of the study products was assessed by interviewing volunteers throughout the study period.

\section{HPLC analysis of atenolol}

Atenolol concentrations in plasma samples were determined after protein precipitation using a sensitive and reproducible HPLC method with fluorometric detection, as described previously $[17,18]$. Metoclopramide was used as the internal standard (IS). All chemicals and reagents were of analytical grade and solvents were of HPLC grade.

An Agilent Laboratories HPLC device, 1200 Series (Santa Clara, CA, USA) was used for analysis. The device was equipped with a Zobrax extend HPLC C18 column $(150 \times 4.6 \mathrm{~mm}$; 5 - $\mu \mathrm{m}$ particle size; Agilent). The isocratic mobile phase consisted of water and methanol (85:15), 0.05\% triethylamine, and was adjusted to $\mathrm{pH} 3$ with phosphoric acid. The flow rate was set at $0.8 \mathrm{~mL} / \mathrm{min}$, and the separated samples were detected using a fluorescence detector maintained at $\lambda_{\mathrm{ex}} 229 \mathrm{~nm}$ and $\lambda_{\mathrm{em}}$ $300 \mathrm{~nm}$.

A stock standard solution of atenolol $(1 \mathrm{mg} / \mathrm{mL})$ was prepared in methanol and a stock IS solution of metoclopramide $(1 \mathrm{mg} / \mathrm{mL})$ was prepared in a methanol: water mixture (1:1). Working standard solutions were prepared by appropriate dilution in methanol. Solutions were protected from light and stored at $-20^{\circ} \mathrm{C}$.

Plasma sample aliquots $(1 \mathrm{~mL})$ were vortex-mixed with $200 \mu \mathrm{L}$ IS [1 $\mathrm{mg} / \mathrm{mL}$ ) and $200 \mu \mathrm{L}$ trichloroacetic acid (TCA); 55\%, final concentration 7.86\%] for $20 \mathrm{~s}$, followed by centrifugation at $3500 \mathrm{rpm}$ for $20 \mathrm{~min}$. The supernatant was filtered using a $0.45-\mu \mathrm{m}$ Millipore filter (EMD Millipore, USA) and $200 \mu \mathrm{L}$ of the filtrate was injected.

Atenolol recovery was calculated by comparing peak areas for standard samples in spiked plasma before and after the preparation procedure. The stability of atenolol in TCA was assessed by comparing chromatograms of atenolol dissolved in either methanol or TCA.

The following parameters were determined: specificity, linearity, accuracy, precision, and limit of quantification. Specificity was checked by comparing chromatograms of blank plasma and plasma samples containing atenolol and the IS following sample preparation. Linearity was assessed by constructing a calibration curve of atenolol in spiked plasma at a concentration range of $25-800 \mathrm{ng} / \mathrm{mL}$. Inter-day accuracy and precision were determined by analysis of spiked plasma samples at three different atenolol concentrations $(50,200$ and $800 \mathrm{ng} / \mathrm{mL}$ ) on three consecutive days. Data for intra-day accuracy and precision were obtained by six replicate analyses carried out on the same day. The relative standard deviation should be within $\pm 15 \%$. The limit of quantification of atenolol was determined by calculating the amount of drug exhibiting a signal:noise ratio of 10:1.

\section{Statistical analysis}

Pharmacokinetic parameters for the two study products were calculated using EquivTest/PK software (Statistical Solutions Unit7B, Farmer's Cross, Ireland). Log transformed means of ratios of the pharmacokinetic parameters maximum plasma concentration $\left(\mathrm{C}_{\max }\right)$, area under the plasma concentration curve from administration to $24 \mathrm{~h}\left(\mathrm{AUC}_{0-24 \mathrm{~h}}\right)$ and AUC extrapolated to infinity $\left(\mathrm{AUC}_{0-\alpha}\right)$ were used to test bioequivalence based on the $90 \%$ confidence interval (CIs) of the ratios. A twoway analysis of variance for crossover design, performed using Minitab Statistical Package, version 15 (State College, PA, USA), was used to assess the effects of treatment, period, sequence, and participants nested within sequence on $\mathrm{C}_{\text {max }}$ and AUC parameters. No carryover or period effects were noted. Paired $t$ tests were carried out for statistical analysis of pharmacodynamic data.

\section{Results}

\section{Pharmacokinetic evaluation}

The recovery of atenolol from plasma samples was 88.3 (SD 6.2)\%, 90.0 (SD $2.9) \%$, and 98.9 (SD 1.7)\% at concentrations of 100,400 , and $800 \mathrm{ng} / \mathrm{mL}$, respectively. Calibration curves for peak area ratios of atenolol in spiked plasma were linear within the concentration range $25-800 \mathrm{ng} / \mathrm{mL}\left(r^{2}=0.997\right)$. The limit of quantification was $25 \mathrm{ng} / \mathrm{mL}$. The coefficient of variation for inter-day and intra-day accuracy and precision data was $<15 \%$. Atenolol was stable in TCA, and no additional peaks were found in chromatograms of atenolol treated with TCA. The retention time of atenolol and metoclopramide (IS) was $7.0 \mathrm{~min}$. and $2.9 \mathrm{~min}$. respectively.

The mean plasma concentrationtime profiles following administration of the reference and test atenolol tablets are shown in Figure 1, and pharmacokinetic parameters are shown in Table 1 . Peak plasma atenolol concentrations 
were attained at a similar time to reach $\mathrm{C}_{\max }\left(\mathrm{t}_{\max }\right)$ of 3.6 (SD 1.20) and 3.6 (SD 1.25) $\mathrm{h}$ for the test and reference products respectively. The test tablets showed a higher arithmetic mean $\mathrm{C}_{\max }$ of 340.2 (SD 198.4) ng/mL compared with 329.29 (SD 151) ng/mL for the reference tablets. Table 1 also shows elimination rate constants $\left(\mathrm{k}_{\mathrm{el}}\right)$ and halflives of the two products. The $90 \%$ CIs of the mean ratios (test/reference) for $\mathrm{C}_{\max }(1.03), \mathrm{t}_{\max }(1.0)$ and $\mathrm{AUC}{ }_{0 \rightarrow 24}$ (0.95) were within 80\%-120\%.

Mean values of ratios (test/reference) of the log-transformed values for $\mathrm{C}_{\text {max }} \mathrm{AUC}_{0 \rightarrow 0^{\circ}}$ and $\mathrm{AUC}_{0 \rightarrow \infty}$ were 101.4 (90\% CI: 92.9-109.9), 99.3 (90\% CI: $92.8-105.7)$ and $98.0(90 \% \mathrm{CI}$ : $88.6-107.3)$ respectively. The differences between these parameters for the test and reference products were not statistically significant. The $90 \% \mathrm{CI}$ values fell within the range $80 \%-125 \%$. According to the FDA guidelines [20], this indicates average bioequivalence of the two study products.

\section{Pharmacodynamic activity}

Blood pressure and heart rate were measured at rest at baseline and following administration of the study products throughout the 24-h blood sampling period. Systolic blood pressure (SBP) and diastolic blood pressure (DBP) reached minimal values at $\sim 5 \mathrm{~h}$. This was followed by a gradual increase at $\sim 8 \mathrm{~h}$ post-dose, although values remained consistently below baseline for up to $24 \mathrm{~h}$. Figure

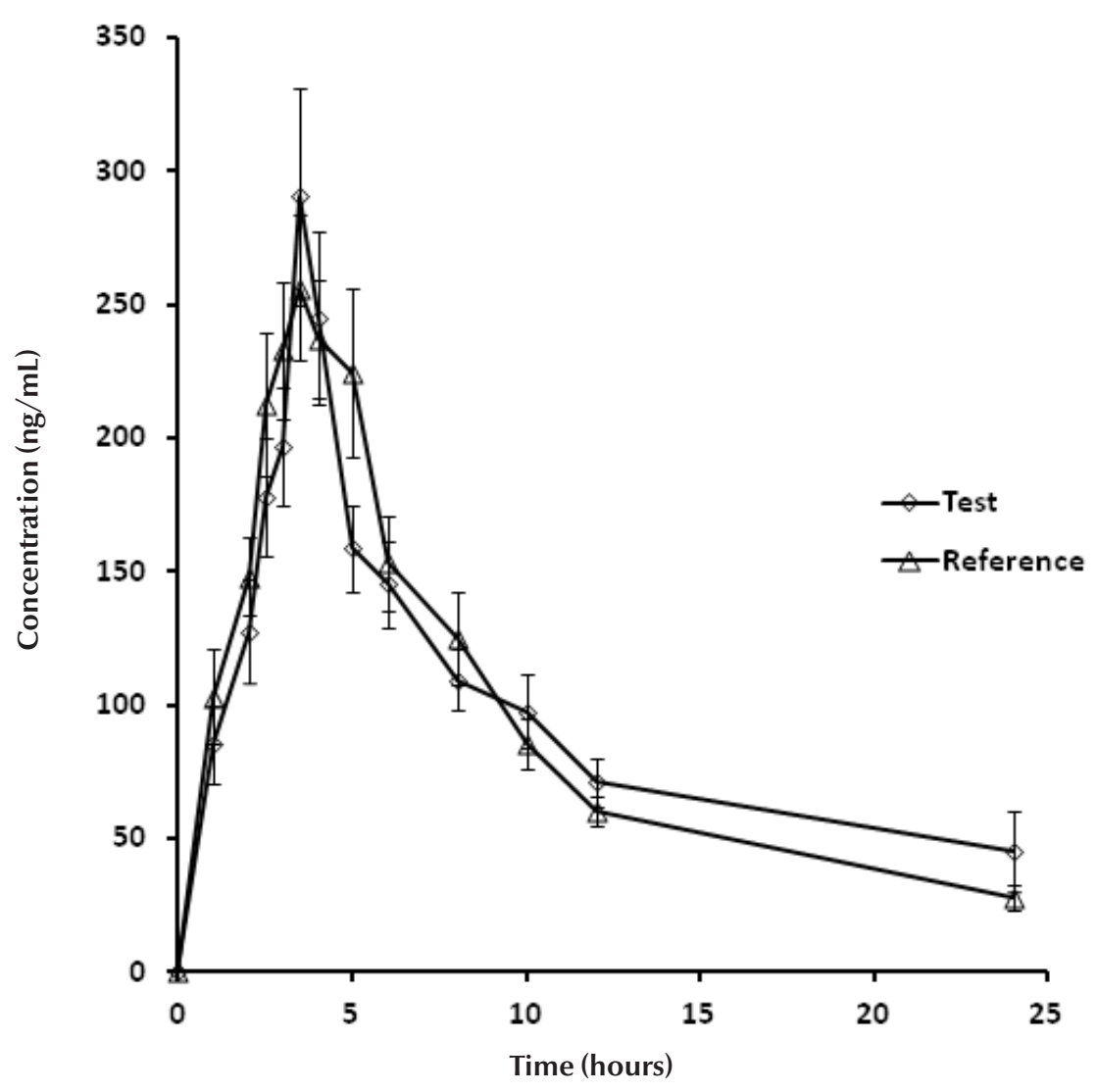

Figure 1 Mean plasma levels of atenolol following administration of 100-mg tablets of the test and reference study products in 24 healthy male subjects (error bars represent standard error of the mean)

2 shows that the time course of blood pressure was similar for the test and reference products. The mean maximum fall in blood pressure for test and reference products was 18.2 SD 14.4 and 13.2 SD $12.1 \mathrm{~mm} \mathrm{Hg}$ for SBP and 14.1 SD 11.4 and $12.0 \mathrm{SD} 7.7 \mathrm{~mm} \mathrm{Hg}$ for DBP, respectively. Heart rate also showed a maximum reduction of 11.7 SD 8.1 and 10.6 SD 9.7 beats/min 5 h after administration of the test and reference products, respectively. The maximum differences in both parameters at $5 \mathrm{~h}$ were statistically significant $(P<0005)$.

Statistical analysis of the pharmacodynamic effects of the test and reference

\begin{tabular}{|c|c|c|}
\hline \multirow[t]{3}{*}{ Pharmacokinetic parameter } & \multicolumn{2}{|c|}{ Treatment } \\
\hline & Test product & Reference product \\
\hline & Mean (SD) & Mean (SD) \\
\hline $\mathrm{AUC}_{0 \rightarrow 24}(\mathrm{ng} \cdot \mathrm{h} / \mathrm{mL})$ & 2021.7 (826.9) & $2123.3(1017.3)$ \\
\hline $\mathrm{AUC}_{0 \rightarrow \infty}(\mathrm{ng} \cdot \mathrm{h} / \mathrm{mL})$ & $2488.7(1235.8)$ & $2625.8(1315.3)$ \\
\hline $\mathrm{C}_{\max }(\mathrm{ng} / \mathrm{mL})$ & $340.1(198.4)$ & $329.3(151.2)$ \\
\hline $\mathrm{T}_{\max }(\mathrm{h})$ & $3.6(1.2)$ & $3.6(1.3)$ \\
\hline$k_{e l}\left(h^{-1}\right)$ & $0.102(0.056)$ & $0.125(0.146)$ \\
\hline$t_{1 / 2}(h)$ & $8.90(4.6)$ & $9.4(5.6)$ \\
\hline
\end{tabular}

$S D=$ standard deviation. 


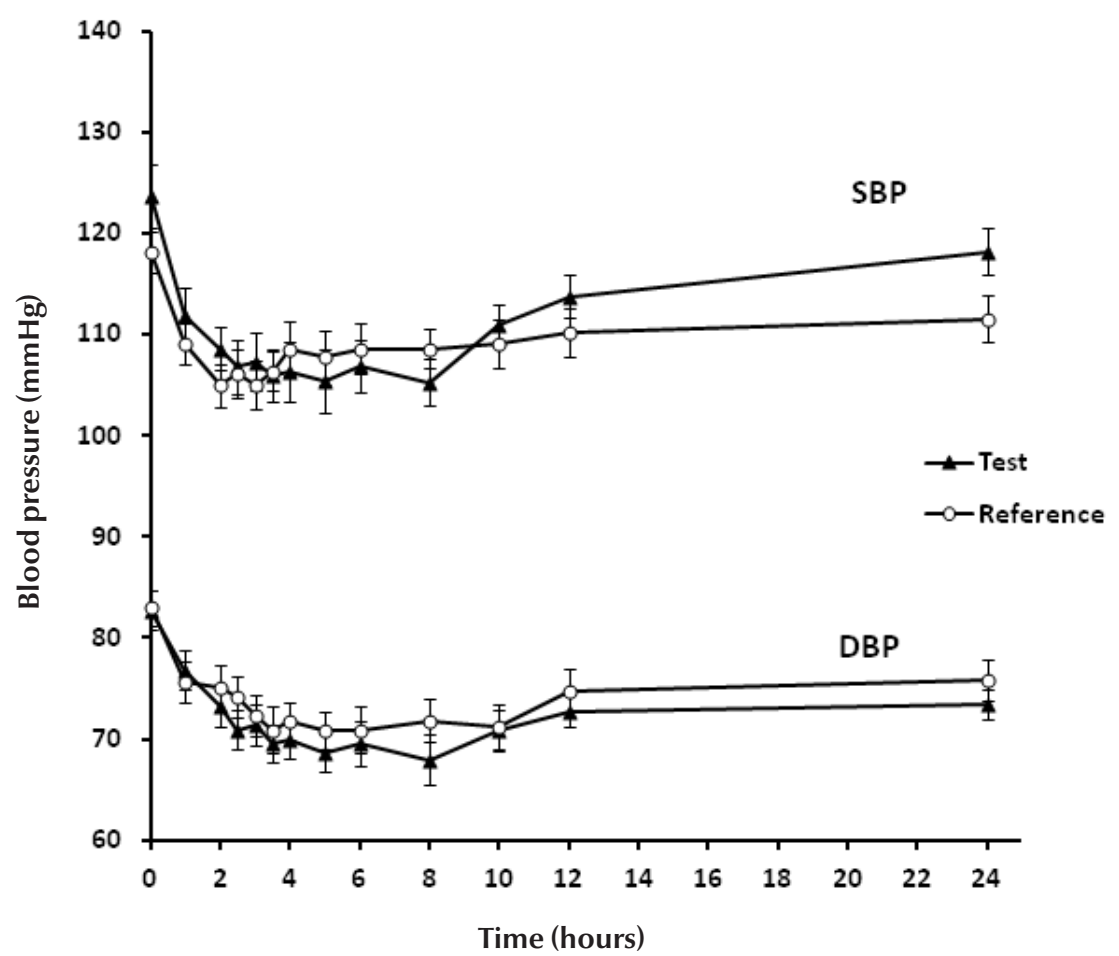

Figure 2 Time course of systolic and diastolic blood pressure (SBP and DBP) following administration of 100-mg tablets of the test and reference study products in 24 healthy male subjects (error bars represent standard error of the mean)

products using the percentage difference between baseline and minimal values indicated that SBP (0.263), DBP (0.568) and heart rate $(0.861)$ were not significantly different $(P>0.05)$.

\section{Adverse effects}

Atenolol was generally well tolerated by all volunteers, except for three with transient mild dizziness and headache, which resolved within a few hours with no medical intervention.

\section{Discussion}

The analytical method used was sensitive and accurate for the assay of atenolol in plasma at all sampling times. The method was based on the precipitation of plasma proteins with TCA as a single sample preparation step. A similar method has been reported recently [21].

$\mathrm{C}_{\max }$ and AUC values of atenolol (Table 1) were lower than those reported in other study populations but readily measured pharmacological endpoint, such as antihypertensive drugs $[18,22,25]$, and have been suggested as part of a more specific study design for investigations in healthy subjects [22-24]. Moreover, combined studies have been used to assess the tolerability of formulations subjected to single dose bioequivalence testing [22,23], and to support bioequivalence data of products of chiral drugs with simple pharmacokinetic profiles such as atenolol [16,18,22] and amlodipine [25].

The $\beta$-blocking activity of the generic product was compared with that of Tenormin, a reference product with reported pharmacodynamic activity in healthy subjects $[16,18,22]$.

SBP, DBP and heart rate were significantly reduced at $\sim 5 \mathrm{~h}$ after administration of atenolol, with gradual recovery towards baseline values. Data obtained were in agreement with those reported for pharmacodynamic effects of a single $100 \mathrm{mg}$ dose of different atenolol tablets formulations in healthy subjects at rest $[15,18,19,22]$. The difference between mean maximal lowering of SBP, DBP and heart rate by both products was not significant. It has been reported that after oral administration of atenolol to healthy normotensive individuals, there were synchronous reductions in blood pressure and heart rate $[16,18,22]$. The pharmacodynamic effects of atenolol in the present study could be qualitatively correlated with blood level data, reported in the manufacturer's Tenormin monograph (Astra Zeneca Pharmaceuticals, Canada, Inc., revised September 2011).

Pharmacokinetic data coupled with pharmacodynamic activity for the test product Ateno and the reference product Tenormin indicated that both products were bioequivalent. They did not differ significantly in pharmacodynamic response in terms of SBP, DBP and heart rate in healthy subjects for up to $24 \mathrm{~h}$ post dosing. According to the WHO [1], these two products can be considered interchangeable in clinical practice. 
The limitations of the study were those recognized for single-dose bioequivalence studies carried out in healthy volunteers. In addition, the generalizability of bioequivalence data obtained in the study to other generic atenolol products on the market and throughout the shelf-life of these products stored under variable conditions is limited.

In conclusion, conformation of pharmacokinetic data to bioequivalence criteria and similar pharmacodynamic activity and tolerability of the generic and reference products provide evidence for equivalence of the two products in the postmarketing setting. The study contributes to the evidence supporting the notion that generic atenolol and other drug products are not less effective than brand-name products, thus enhancing confidence of the local clinical community in generics. Finally, the independent postmarketing reassessment approach adopted in this study can be considered for responding to patient and clinician complaints regarding product interchangeability. This may be of value in promoting safety of medicines for the general public and informed planning of drug use.

Funding: This work is part of a research project funded by the Alexandria University Research Enhancement Program (ALEX REP), Egypt. Project Code: BEPH1.

Competing interests: None declared.

\section{References}

1. World Health Organization. Multisource (generic) pharmaceutical products: guidelines on registration requirements to establish interchangeability. In: WHO Expert Committee on Specifications for Pharmaceutical Preparations. WHO Technical Report Series number 937, Fortieth report, 2006, Annex 7:347-390.

2. Bahgat $H$, Wright R. Access to medicines in Egypt: a human rights approach to intellectual property, trade and health. In: Risk N, Shaver L, eds. Access to knowledge in Egypt. New research on intellectual property, innovation and development. London, New York, Bloomsbury Academic, 2010:56-91.

3. The pharmaceutical market: Egypt. ESPICOM Business Intelligence, June 2011.

4. Chen ML et al. Harmonization of regulatory approaches for evaluating therapeutic equivalence and interchangeability of multisource drug products: workshop summary report. European Journal of Pharmaceutical Sciences, 2011, 44:506-513.

5. Kameron G. The pros and cons of generic drugs. BMJ 2011, 343:d4584.

6. Crawford $\mathrm{P}$ et al. Are there potential problems with generic substitution of antiepileptic drugs? A review of issues. Seizure, 2006, 15:165-176.

7. Meredith P. Bioequivalence and other unresolved issues in generic drug substitution. Clinical Therapeutics, 2003, 25:2875-2890.

8. Del Tacca M et al. Lack of pharmacokinetic bioequivalence between generic and branded amoxicillin formulations. A post-marketing clinical study on healthy volunteers. British Journal of Clinical Pharmacology, 2009, 68:34-42.

9. Lopes RA, Neves FR. Meta-analysis for bioequivalence studies: interchangeability of generic drugs and similar containing hydrochlorothiazide is possible but not for those with enalapril maleate. Jornal Brazileiro de Nefrologia, 2010, 32:173-181

10. Elkoshi Z, Behr D, Mirimsky A. Multiple-dose studies can be a more sensitive assessment for bioequivalence than single-dose studies: the case with omeprazole. Clinical Drug Investigation, 2002, 22:585-592.

11. Risha, PG et al. Drug formulations intended for the global market should be tested for stability under tropical climatic conditions. European Journal of Pharmaceutical Sciences, 2003, 59:135-141.

12. WHO essential medicines list, 16th ed. Geneva, World Health Organization, 2010:18.
13. Bangalore $\mathrm{S}$ et al. Cardiovascular protection using betablockers. Journal of the American College of Cardiology, 2007, 50:563-572.

14. Mehvar R. Stereospecific pharmacokinetics and pharmacodynamics of beta adrenergic blockers in humans. Journal of Pharmacy and Pharmaceutical Sciences, 2001, 4:185-200.

15. Clementi WA et al. Single dose pharmacokinetics of (S)-atenolol administered orally as a single enantiomer formulation and as a racemic mixture (Tenormin ${ }^{\mathrm{TM}}$ ). Chirality, 1994, 6:169-174.

16. Sarkar MA et al. Pharmacodynamic and pharmacokinetic comparisons to evaluate bioequivalence of atenolol. Clinical Research and Regulatory Affairs, 1995, 12:47-62.

17. Najib NM et al. Comparative bioavailability of two brands of atenolol. Biopharmaceutics and Drug Disposition, 2005, 26:1-5.

18. Mifrazaelian A et al. Bioequivalence study of atenolol: pharmacokinetic and pharmacodynamic evaluation. DARU, 2003, 11:1-4.

19. Chiang HT et al. A comparison of antihypertensive effects between two formulations of atenolol: Tenolol and Tenormin. Chinese Medical Journal, 1995, 55:366-370.

20. FDA. Guidelines for industry: bioavailability and bioequivalence studies for orally administered drug products - general considerations. Rockville, MD, FDA Center for Drug Evaluation and Research, 2003.

21. Elgawish MS, Mostafa SM, Elshanawane AA. Simple and rapid HPLC method for simultaneous determination of atenolol and chlorthalidone in spiked human plasma. Saudi Pharmaceutical Journal, 2011, 19:43-49.

22. Cuadrado A et al. In vitro and in vivo equivalence of two oral atenolol tablet formulations. Arzneimittelforschung, 2002, 52:371-378.

23. Queckenberg $\mathrm{C}$ et al. Pharmacokinetics, pharmacodynamics, and comparative bioavailability of single, oral 2-mg doses of dexamethasone liquid and tablet formulations: a randomized, controlled, crossover study in healthy adult volunteers. Clinical Therapeutics, 2011, 33:1831-1841.

24. Czendlik $\mathrm{CH}$ et al. Pharmacokinetic and pharmacodynamic interaction of single doses of valsartan and atenolol. European Journal of Clinical Pharmacology, 1997, 52:451-459.

25. Park JY et al. Pharmacokinetic and pharmacodynamic characteristics of a new $S$-amlodipine formulation in healthy Korean male subjects: a randomized, open-label, two-period, comparative, crossover study. Clinical Therapeutics, 2006, 28:1837-1847. 